\title{
Remote Activation of Hollow Nanoreactors for Heterogeneous Photocatalysis in Biorelevant Media
}

Ana Sousa-Castillo, José R. Couceiro, María Tomás-Gamasa, Andrea MariñoLópez, Fernando López, Walid Baaziz, Ovidiu Ersen, Miguel Comesaña-Hermo, José L. Mascareñas, Miguel A. Correa-Duarte

\section{Peer reviewed version}

This is the peer reviewed version of the following article: Sousa-Castillo, A.; Couceiro, J. R.; Tomás-Gamasa, M.; Mariño-López, A.; López, F.; Baaziz, W.; Ersen, O.; ComesañaHermo, M.; Mascareñas, J. L.; Correa-Duarte, M. A. (2020), Remote Activation of Hollow Nanoreactors for Heterogeneous Photocatalysis in Biorelevant Media. Nano Lett., 20: 7068-7076, which has been published in final form at https://doi.org/10.1021/acs.nanolett.0c02180. This article may be used for noncommercial purposes in accordance with ACS Terms and Conditions for Use of SelfArchived Versions.

\section{How to cite:}

Sousa-Castillo, A.; Couceiro, J. R.; Tomás-Gamasa, M.; Mariño-López, A.; López, F.; Baaziz, W.; Ersen, O.; Comesaña-Hermo, M.; Mascareñas, J. L.; Correa-Duarte, M. A. (2020), Remote Activation of Hollow Nanoreactors for Heterogeneous Photocatalysis in Biorelevant Media. Nano Lett., 20: 7068-7076. doi: 10.1021/acs.nanolett.0c02180

\section{Copyright information:}

(C) 2020 ACS. This article may be used for non-commercial purposes in accordance with ACS Terms and Conditions for Use of Self-Archived Versions 


\section{Remote Activation of Hollow Nanoreactors for Heterogeneous}

\section{Photocatalysis in Bio-relevant Media}

Ana Sousa-Castillo, ${ }^{\dagger}$ José R. Couceiro, ${ }^{\ddagger}$ María Tomás-Gamasa, ${ }^{\ddagger}$ Andrea Mariño-López, ${ }^{\dagger}$ Fernando López,,$\$$ Walid Baaziz, ${ }^{\S}$ Ovidiu Ersen, ${ }^{\S}$ Miguel Comesaña-Hermo, ${ }^{\|, *}$ José L. Mascareñas, ${ }^{\ddagger} * *$ Miguel A. Correa-

$$
\text { Duarte }^{\dagger, \delta, *}
$$

${ }^{\dagger}$ CINBIO, Universidade de Vigo, As Lagoas, Marcosende, 36310 Vigo, Spain

${ }^{8}$ Southern Galicia Institute of Health Research (IISGS), and Biomedical Research Networking Center for Mental Health (CIBERSAM), Spain

*Centro Singular de Investigación en Química Biolóxica e Materiais Moleculares (CIQUS), Departamento de Química Orgánica, Universidade de Santiago de Compostela, 15782 Santiago de Compostela, Spain

"Instituto de Química Orgánica General (IQOG), CSIC, Juan de la Cierva 3, 28006, Madrid, Spain

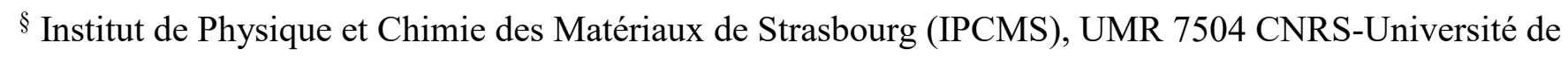
Strasbourg (UdS), 23 rue du Loess, 67037 Strasbourg Cedex 08, France

' Université de Paris, ITODYS, CNRS, F-75006 Paris, France

Corresponding authors: miguel.comesana-hermo@u-paris.fr, joseluis.mascarenas@usc.es,macorrea@uvigo.es, 


\begin{abstract}
Major current challenges in nanobiotechnology and nanobiomedicine include the implementation of predesigned chemical reactions in biological environments. In this context, heterogeneous catalysis is emerging as a promising approach to extend the richness of organic chemistry onto the complex environments inherent to living systems. Herein we report the design and synthesis of hybrid heterogeneous catalysts capable of being remotely activated by NIR light for the performance of selective photocatalytic chemical transformations in biological media. This strategy is based on the synergistic integration of $\mathrm{Au}$ and $\mathrm{TiO}_{2}$ nanoparticles within mesoporous hollow silica capsules, thus permitting an efficient hot-electron injection from the metal to the semiconductor within the interior of the capsule that leads to a confined production of reactive oxygen species. These hybrid materials can also work as smart NIR-responsive nanoreactors inside living mammalian cells, a cutting-edge advance towards the development of photoresponsive theranostic platforms.
\end{abstract}

\title{
Keywords
}

Photocatalysis; bioorthogonal chemistry; plasmonic hot electrons; hybrid nanocapsules 
Titanium dioxide $\left(\mathrm{TiO}_{2}\right)$ stands out among the inorganic semiconductors due to its high abundance, low cost, chemical stability and unique photochemical and photoelectrochemical properties. ${ }^{1}$ Owing to these characteristics, $\mathrm{TiO}_{2}$-based photocatalysts have been exploited in many applications ranging from environmental purifications to bactericidal uses. A major drawback in further extending the applicability of $\mathrm{TiO}_{2}$ as photoresponsive material is associated to its wide bandgap $(3.2 \mathrm{eV})$, which restricts its photocatalytic capabilities to the ultraviolet (UV) segment of the solar radiation spectrum. ${ }^{2}$ Accordingly, the well-known sensitivity of many biomolecules to UV light impedes the use of $\mathrm{TiO}_{2}$ in biological contexts. ${ }^{3}$

Hybrid nanomaterials made by the combination of plasmonic metals $\left(\mathrm{Au}, \mathrm{Ag}\right.$ or $\mathrm{Cu}$ ) and $\mathrm{TiO}_{2}$ allow to extend the photoresponse of the semiconductor to the visible and near-infrared (NIR) regions of the electromagnetic spectrum. ${ }^{4,5}$ Upon irradiation, the plasmonic nanoparticles (NPs) generate hot electrons which are transferred to the semiconductor to produce an efficient separation of charges. This state, with an excited (hot) electron in the conduction band of the semiconductor and a hole in the Fermi level of the plasmonic metal, can be very effective to promote reduction or oxidation reactions. ${ }^{6}$ Indeed, the synergistic effects arising when combining both species have already been implemented in several photocatalytic applications such as water splitting, ${ }^{7}$ pollutant degradation, ${ }^{8} \mathrm{CO}_{2}$ reduction ${ }^{9}$ or oxidation reactions. ${ }^{10}$ The low energy radiation associated to the excitation of these heterogeneous reactors would overcome the intrinsic optical limitations of large bandgap semiconductors such as $\mathrm{TiO}_{2}$, thus providing for biological use. In this context, the development of photocatalytic biocompatible and bioorthogonal reactions is especially appealing, as it would forge to a completely new field of research. ${ }^{11-13}$ Interestingly, such approach is radically different from the photothermal processes currently under investigation in plasmonics, in which the optical excitation of the metal produces a localized temperature increase that can be implemented in biological environments, finding applications in catalysis, ${ }^{14}$ hyperthermia ${ }^{15}$ or sensing. ${ }^{16}$ Herein we provide the first examples on the use of NIR photoresponsive nanocomposites to promote specific reactions of organic precursors in biorelevant media. In particular, we report the design and preparation of hybrid nanocomposites featuring $\mathrm{Au}$ nanorods (NRs) and $\mathrm{TiO}_{2} \mathrm{NPs}$, synergistically integrated within a mesoporous hollow silica capsule. The design of these materials stems from the notion of protecting the nanocatalyst inside the capsule from potentially harmful components present in biological environments, ${ }^{17}$ while ensuring the 
confined production of reactive oxygen species (ROS) in their inner cavity. This unique architecture allows the selective chemical transformation of the desired substrates within the inner space of the mesoporous reactor by means of NIR radiation, extending the usability of the hybrid nanocatalyst to complex biological media. As a proof of concept, preliminary experiments indicate the suitability of this reactors to perform the transformation of a fluorescent substrate in living cells.

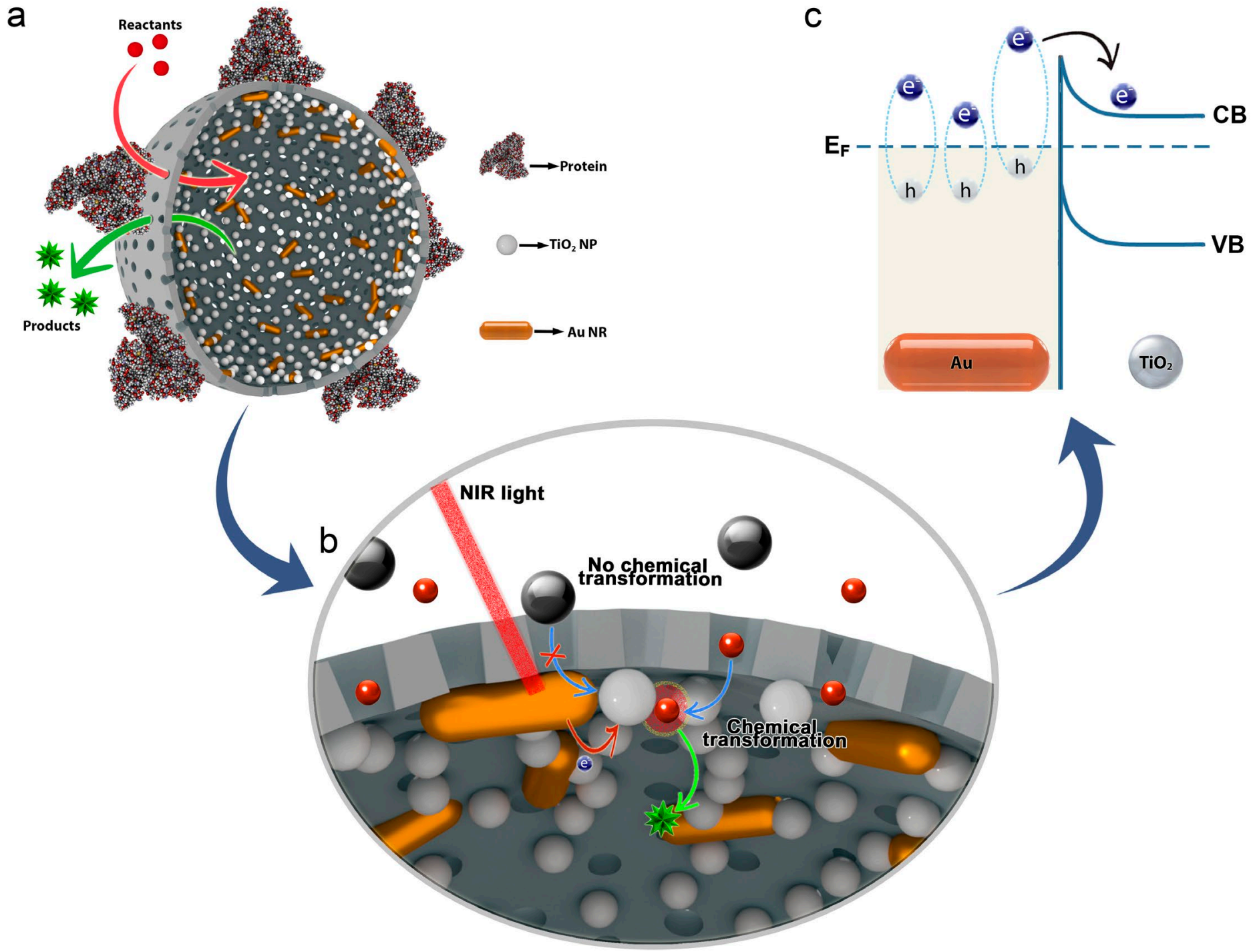

Scheme 1. (a) Illustration depicting the architecture of the proposed nanoreactors $\left(\mathrm{Au}-\mathrm{TiO}_{2} \mathrm{NCs}\right)$. (b) Detail of the chemical transformation taking place at the internal cavity of the mesoporous silica capsules. Red spheres represent molecules diffusing inside the cells, while black spheres remain outside because of steric bulkiness. (c) Hot electron injection mechanism between the Fermi level of the metal and the conduction band of the semiconductor. 
As already mentioned, key for the success of the approach is the design of biocompatible nanostructures that protect the $\mathrm{Au}-\mathrm{TiO}_{2}$ nanoreactors from the biomolecules and salts present in biological buffers, hence ensuring the hot electron injection in the metal-semiconductor hybrids and the subsequent electron exchanging processes with the reactants (Scheme 1). In order to tackle this issue, we prepared hollow mesoporous silica nanocapsules (NCs) whose internal walls have been functionalized with $\mathrm{TiO}_{2}$ NPs and $\mathrm{Au}$ NRs $\left(\mathrm{Au}-\mathrm{TiO}_{2} \mathrm{NCs}\right.$, Scheme 1a). The combination of titania with anisotropic plasmonic NPs is known to be an exceptional strategy to increase the photocatalytic properties of the former. ${ }^{8,18}$ Previous studies have shown that the extent of photosensitization depends on a number of factors such as the chemical composition and shape anisotropy of the plasmonic NP, the energy difference between the conduction band of the semiconductor and the Fermi level of the metal or the quality of the physical interface created between both materials. ${ }^{19}$ Along these lines, the relatively small energy gap between the conduction band of $\mathrm{TiO}_{2}$ and the Fermi level of $\mathrm{Au}(\approx 1 \mathrm{eV})$ allows for an efficient electron injection across the Schottky barrier (Scheme 1c). We chose Au as plasmonic photosensitizer due to its higher chemical stability when compared to other elements such as $\mathrm{Ag}$ or $\mathrm{Cu}$ and because Au nanostructures present a plasmonic response that can be easily tuned throughout the visible and NIR regions of the electromagnetic spectrum. ${ }^{20}$ In that sense, the use of anisotropic Au NPs as photosensitizing agents would ensure a main absorption contribution within the first biological transparency window of the NIR range $(650-950 \mathrm{~nm}){ }^{21,22}$ The morphology of the hybrid nanostructure and the chemical composition of its outermost shell are two key characteristics for the correct design of a final composite with the desired properties and functionalities. In this vein, the encapsulation of the photoactive components within the internal walls of a void silica NC presents several advantages. ${ }^{23,24}$ Firstly, the mesoporous character of the silica shell should permit the control of molecular traffic in and out of the cavity, allowing size selectivity while keeping the semiconductor and plasmonic components away from an eventual non-specific binding of biomolecules. ${ }^{16,25}$ In a similar way, the ROS formed after activation of the semiconductor are unable to diffuse out of the nanostructure given their short lifetimes, thus limiting their activity to the inner compartment within the nanohybrid. Moreover, the low cytotoxicity, easy functionalization and high chemical stability of silica should warrant a high biocompatibility. ${ }^{26,27}$ Finally, the encapsulation of $\mathrm{Au}$ NRs inside the silica shell endows these anisotropic objects with an improved photostability, 
preventing the reshaping often suffered by this type of NPs when exposed to high intensity radiation sources such as laser pulses. ${ }^{28}$ Overall, the engineered hybrid material gathers all the necessary characteristics to expand the application of the photocatalytic properties of bare $\mathrm{TiO}_{2}$, especially in biological contexts.

The synthesis of the proposed capsules $\left(\mathrm{Au}-\mathrm{TiO}_{2} \mathrm{NCs}\right)$ was carried using a multistep process in which the different components that form the final hybrid are added in a sequential fashion (Figure 1a-h) ${ }^{29}$ Firstly, polystyrene (PS) beads with a mean diameter of $500 \mathrm{~nm}$ are functionalized with a layer of positively-charged poly(allylamine hydrochloride) (PAH), allowing the consecutive deposition of $\mathrm{TiO}_{2} \mathrm{NPs}$ and $\mathrm{Au}$ NRs following the well-known layer-by-layer assembly protocol. ${ }^{30}$ Such methodology allows the homogeneous distribution of $\mathrm{TiO}_{2} \mathrm{NPs}_{\text {s }}$ onto the PS substrate while ensuring an intimate contact between the semiconductor and Au NRs (Figure S1), essential to ensure an efficient electron transfer between them. Subsequently, a CTAB-templated mesoporous silica shell is grown onto the PS@Au- $\mathrm{TiO}_{2} \mathrm{NPs}$, leading to the formation of a homogeneous silica coating with a thickness of $25( \pm 3) \mathrm{nm}$ (Figure 1e,f and S2). In the last step, THF is used to remove the CTAB molecules and the polystyrene template, forming a hollow mesoporous silica shell whose internal cavity is functionalized with the semiconductor and the metal components (for more details, please refer to the Supporting Information). The 3D reconstruction of the final objects obtained by electron tomography provides information about the spatial distribution of the photoactive species confined within the internal cavity of the mesoporous structure. As can be seen in Figure 1i, both, the $\mathrm{TiO}_{2}$ NPs and Au NRs, are indeed adsorbed onto the inner wall of the silica shell thus presenting available surfaces to get in contact with the substrates. Importantly, no modification in the morphology of the objects was observed at the end of the synthetic procedure, highlighting the suitability of THF to remove the organic materials in opposition to a calcination process that could otherwise compromise the integrity of the nanocomposites through reshaping and sintering processes. ${ }^{29}$ TEM also allows the visualization of the ordered nanometric pores within the silica shell, characteristic of outmost relevance for the selective diffusion of molecular species into the internal cavity of these structures (Figure $1 \mathrm{j}) .{ }^{25}$

The optical properties of the final composite were characterized by UV-visible-NIR absorption spectroscopy (Figure 1k). At low wavelengths, strong extinction of the nanoreactor is observed, coming from a combination 
of the bandgap excitation of $\mathrm{TiO}_{2} \mathrm{NPs}$ and the scattering contribution of the mesoporous silica shell. More importantly, $\mathrm{Au}-\mathrm{TiO}_{2} \mathrm{NCs}$ maintain the main features of $\mathrm{Au}$ NRs in solution, with their longitudinal plasmon resonance centered at $870 \mathrm{~nm}$, thus slightly red shifted with respect to that of the isolated nanorods $(860 \mathrm{~nm})$. In this context, the material presented herein holds the optical features required for an efficient plasmonic excitation in a biological setting, since the main absorption signature remains in the first biological transparency window.
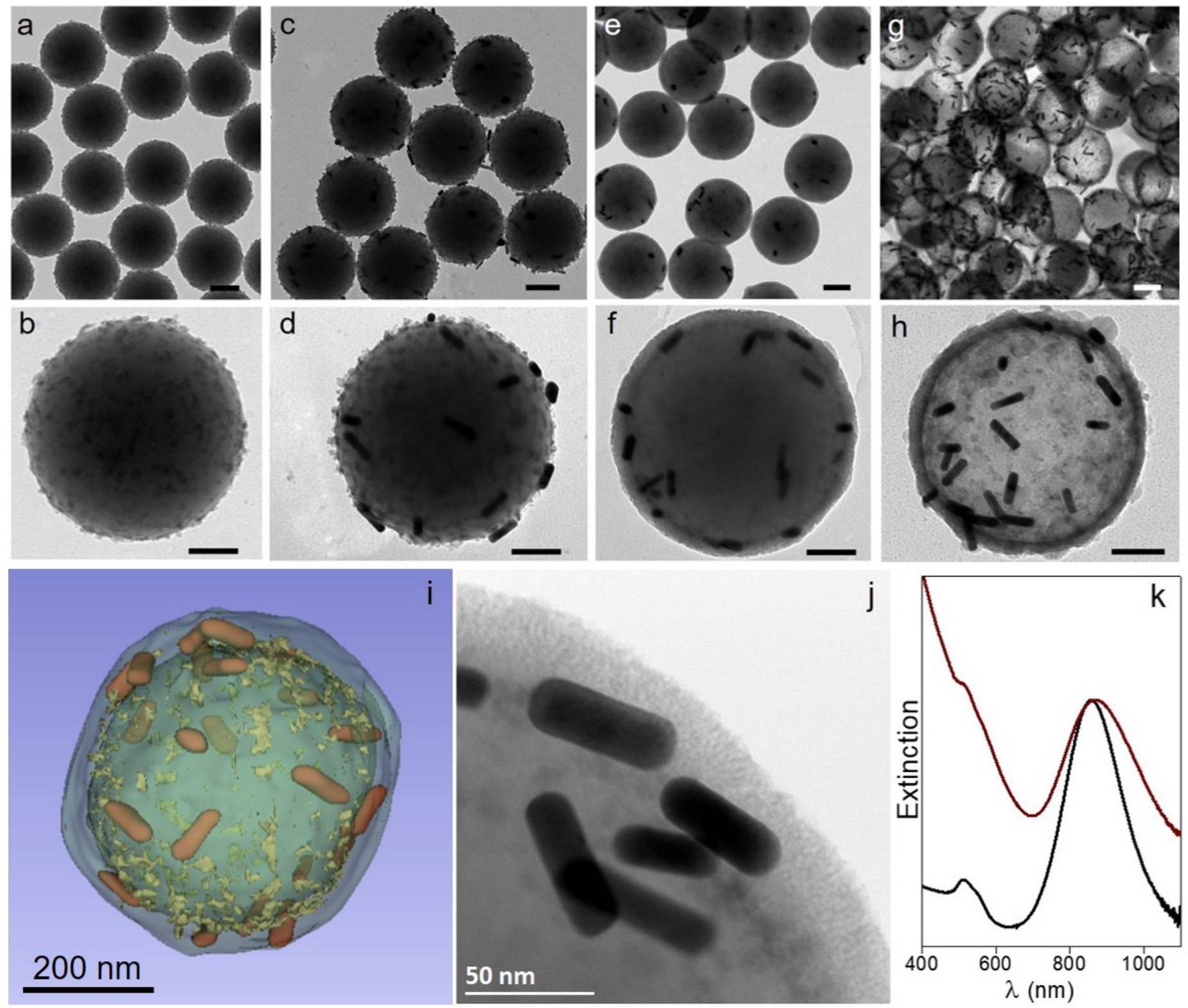

Figure 1. TEM images of different stages of the synthetic procedure: $(\mathrm{a}, \mathrm{b})$ adsorption of $\mathrm{TiO}_{2} \mathrm{NPs}$ on PS beads, (c, d) adsorption of $\mathrm{Au}$ NRs, (e, f) mesoporous $\mathrm{SiO}_{2}$ coating and (g, h) removal of the PS core and the CTAB molecules present in the silica. Scale bars: $200 \mathrm{~nm}$ in the upper panel and $100 \mathrm{~nm}$ in the lower one. (i) 3D electron tomography reconstruction of the $\mathrm{Au}-\mathrm{TiO}_{2} \mathrm{NCs}$ where the $\mathrm{TiO}_{2} \mathrm{NPs}$ are represented in yellow and the $\mathrm{Au} \mathrm{NRs}$ in 
red. (j) TEM image of the $\mathrm{Au}-\mathrm{TiO}_{2} \mathrm{NCs}$ in which the mesoporous character of the silica can be clearly discerned.

(k) Extinction spectra of $\mathrm{Au}$ NRs (black line) and the $\mathrm{Au}-\mathrm{TiO}_{2} \mathrm{NCs}$ (brown line).

The photocatalytic capabilities of the $\mathrm{Au}-\mathrm{TiO}_{2} \mathrm{NCs}$ were explored in aqueous solutions, using aromatic dyes as substrates. Among the different polycyclic aromatic molecules used as fluorescent dyes for industrial applications, rhodamine $\mathrm{B}(\mathrm{RhB})$ has been previously reported to undergo molecular degradation when exposed to solar radiation in the presence of hybrid $\mathrm{Au}-\mathrm{TiO}_{2}$ nanosystems. This transformation has been chosen as initial reference for our studies because it can be easily tracked by using absorption and fluorescence spectroscopies. ${ }^{18}$ We found that the irradiation of an aqueous solution of $\operatorname{RhB}\left(10^{-5} \mathrm{M}\right)$ with a solar simulator $\left(\lambda_{\text {exc }}: 350-2400 \mathrm{~nm}\right)$, in the presence of the $\mathrm{Au}-\mathrm{TiO}_{2} \mathrm{NCs}$ leads to a $82 \%$ loss in the original absorption signature after 210 min (Figure 2a, for more details refer to the Supporting Information), also accompanied by the disappearance of the fluorescence signal ( $\lambda_{\text {exc }}: 554 \mathrm{~nm}, \lambda_{\text {em }}: 580 \mathrm{~nm}$, Figure $2 \mathrm{~b}$ ). While previous studies in $\mathrm{TiO}_{2}$-promoted photoreactions of RhB and other dyes refer to degradation and even mineralization events, a limited number of them pay attention to the mechanistic insights and the intermediate products obtained in the process. ${ }^{31} \mathrm{~A}$ careful analysis of the products resulting from the photocatalytic reaction of $\mathrm{RhB}$ confirmed that the observed decrease in absorption is associated to de-ethylation reactions (Figure $2 \mathrm{c}-\mathrm{e}$ ). Indeed, the decrease in the absorbance at $\lambda_{\max }$ : $554 \mathrm{~nm}$ (Figure 2a) and fluorescence intensity (Figure 2b) observed upon irradiation are accompanied by the appearance of a new absorption band at shorter wavelengths consistent with the formation of the de-ethylated derivatives such as rhodamine 110 (Rh110), holding an absorption maximum at $499 \mathrm{~nm}$ (black absorption spectrum in Figure 2a). Additional analysis of the reaction media by liquid chromatography corroborates an efficient conversion of RhB into Rh110 under solar simulator irradiation in the presence of the $\mathrm{Au}_{-} \mathrm{TiO} \mathrm{N}_{2} \mathrm{NCs}$ (Figure 2c,d). Furthermore, the evolution of this transformation could be confirmed by mass spectrometry through the detection of the different intermediates involved in the process (Figure 2e).

The photodegradation capabilities of the hybrid capsules are intimately related to their ability to photogenerate ROS. Along these lines, we have performed control experiments in order to shade some light on the nature of these unstable species responsible for the photodegradation of the organic dye. ${ }^{32}$ In this sense, hydroxyl $\left({ }^{\circ} \mathrm{OH}\right)$ 
and superoxide $\left(\mathrm{O}_{2}^{-*}\right)$ radicals have been detected thanks to their specific reaction with two radical scavengers: terephthalic acid and 1,4-benzoquinone probes, respectively (Figure S3). While $\mathrm{O}_{2}^{-{ }^{-}}$is the result of the interaction of hot electrons with $\mathrm{O}_{2}$ molecules, ${ }^{\circ} \mathrm{OH}$ may be formed after interaction of hot holes with water molecules or through the decomposition of photogenerated $\mathrm{H}_{2} \mathrm{O}_{2}$.

In order to ascertain the importance of the hot electron injection between the plasmonic material and the semiconductor, we have compared the photochemical performance of the $\mathrm{Au}-\mathrm{TiO}_{2} \mathrm{NCs}$ with those of silica $\mathrm{NCs}$ whose internal walls are functionalized only with $\mathrm{TiO}_{2} \mathrm{NPs}\left(\mathrm{TiO}_{2} \mathrm{NCs}\right)$ or $\mathrm{Au}$ NRs (AuNCs), respectively (Figure S4). In this manner, the use of $\mathrm{TiO}_{2} \mathrm{NCs}$ leads to the partial degradation of the organic dye (61\%) (Figure $2 \mathrm{~h}$, black line). The observed reactivity can be explained in terms of the direct activation of the semiconductor with high energy photons coming from the UV segment of the simulated solar spectrum. Under the same irradiation conditions, the use of AuNCs does not lead to the degradation of the organic dye. Actually, a small increase in the concentration of $\mathrm{RhB}$ is observed in this case (Figure S5), a result that might be consequence of a higher diffusion of $\mathrm{RhB}$ molecules that were presumably blocked within the silica pores, owing to the temperature increase induced by heat dissipation upon plasmonic excitation.

In order to ascertain the capabilities of the hybrid NCs to discern among different substrates (orthogonality), we compared the photodegradation of $\mathrm{RhB}$ with that of evans blue (EB), another aromatic dye that can be photocatalytically transformed under simulated solar light. EB presents a larger size than $\mathrm{RhB}$, characteristic that may hinder its fluent diffusion through the silica pores (Figure S6). ${ }^{33}$ Previous experiments with similar mesoporous silica capsules have shown that size selectivity is a major parameter directing the reactivity. ${ }^{29}$ Indeed, while $\mathrm{RhB}$ shows a $82 \%$ decrease in its original concentration after $210 \mathrm{~min}$ of irradiation with the solar simulator, EB only presents a reduction of $12 \%$ (Figure $2 \mathrm{f}$ ). The remarkable difference in chemical reactivity is consistent with a size-selective molecular diffusion through the mesoporous channels of the nanoreactors $\left(\mathrm{Au}-\mathrm{TiO}_{2} \mathrm{NCs}\right) .{ }^{29}$ Importantly, when both dyes are irradiated in the presence of "naked" $\mathrm{Au}-\mathrm{TiO}_{2}$ catalysts (i.e. PS beads functionalized with $\mathrm{TiO}_{2}$ NPs and Au NRs, as in Figure 1c,d), which lack the sieving effect capabilities provided by the mesoporous silica shell, both molecules present the same degree of degradation $(\approx 28 \%$, Figure $2 \mathrm{~g})$. These 
results underline the benefits of the capsular configuration of the nanoreactors with respect to the non-hollowed structures. Actually, the difference in efficiency is surely related to the confinement effect provided by the container, hence facilitating the presence of dye molecules near the internal wall of the silica shell, and thus their interaction with ROS created at the nearby metal-semiconductor interface. Even though steric hindrance is the main parameter controlling molecular diffusion within the silica pores, other properties, such as charge distribution or local changes in ionic concentration may also participate in the control of molecular traffic. ${ }^{34}$ Therefore, our $\mathrm{Au}-\mathrm{TiO}_{2} \mathrm{NCs}$ allow to confine the photocatalytic activity to their inner void space while providing for size selective reaction capabilities. This can be especially useful in biological media by filtering the uptake of biomolecules that might poison the reacting core.

We next studied the photochemical reactivity of the $\mathrm{Au}-\mathrm{TiO}_{2} \mathrm{NCs}$ under NIR irradiation. As postulated previously, the direct photosensitization of the semiconductor with the solar simulator leads to a substantial degradation of $\mathrm{RhB}\left(82 \%\right.$ for the $\mathrm{Au}-\mathrm{TiO}_{2} \mathrm{NCs}$ vs. $61 \%$ for the $\mathrm{TiO}_{2} \mathrm{NCs}$, Figure $2 \mathrm{~h}$ ). Importantly, when we use a colored filter that blocks all radiation with $\lambda<700 \mathrm{~nm}$, therefore restricting it to the NIR region of the simulated solar spectrum, the photocatalytic activity of $\mathrm{TiO}_{2} \mathrm{NCs}$ vanishes while the efficiency of the $\mathrm{Au}-\mathrm{TiO}_{2} \mathrm{NCs}_{\text {remains }}$ very high (76\% conversion, Figure $2 \mathrm{i})$. Previous works have shown that the high efficiency under NIR excitation is the result of the predominant role played by the longitudinal plasmon band of $\mathrm{Au}$ NRs in the generation of excited carriers, in our case centered at $870 \mathrm{~nm} .{ }^{8}$ In this manner, the plasmonic photosensitization of $\mathrm{TiO}_{2}$ through hot electron injection ensures the chemical transformations in the inner space of the hybrid reactor under NIR radiation, an important asset when aiming at the development of biocompatible photocatalysts. ${ }^{32}$ 

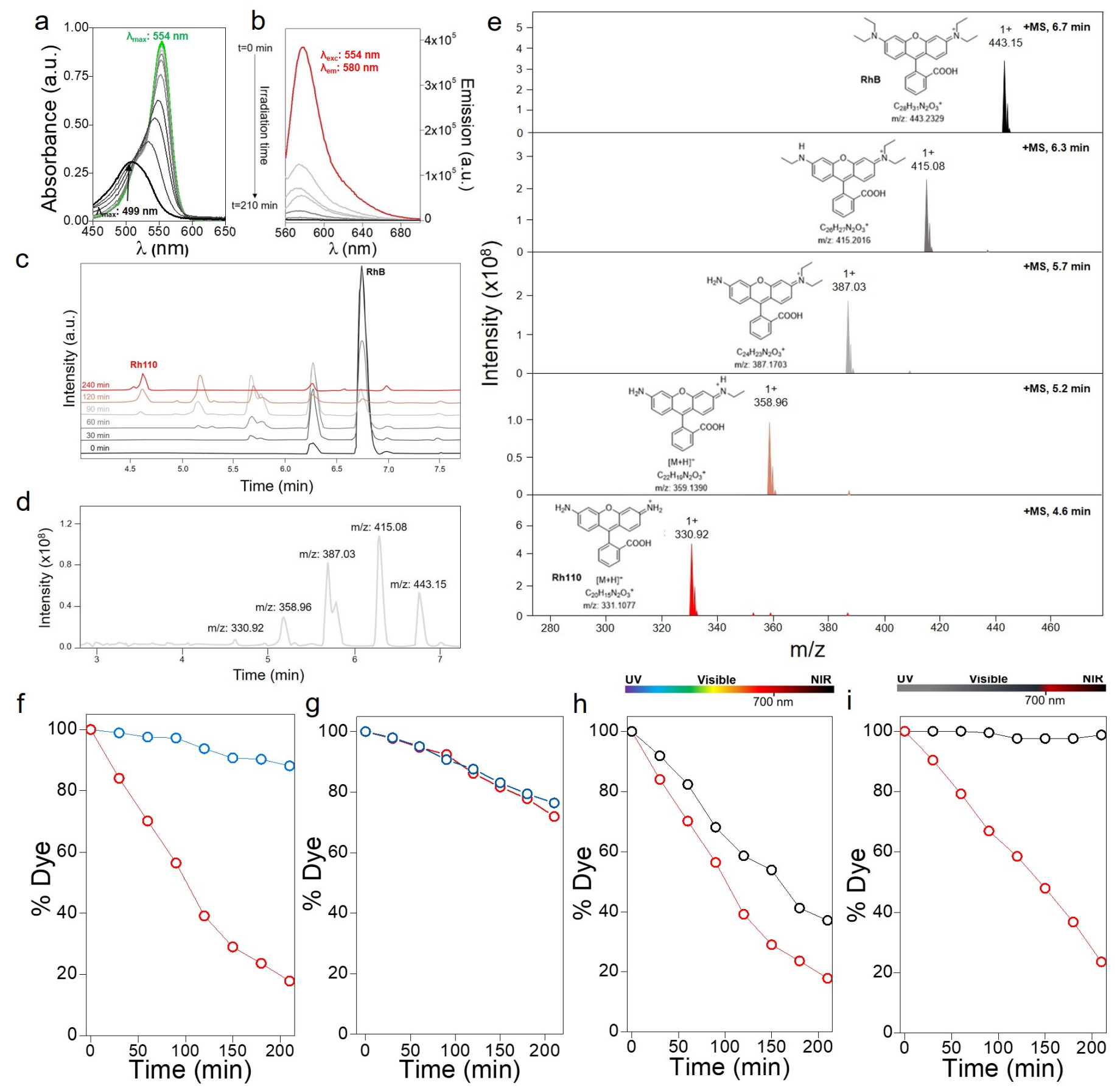

Figure 2. Evolution of the UV-visible absorption (a) and emission (b) spectra of RhB upon irradiation with a solar simulator $(350-2400 \mathrm{~nm})$ in the presence of the $\mathrm{Au}-\mathrm{TiO}_{2} \mathrm{NCs}\left(\lambda_{\text {exc }}\right.$ for the emission spectra: $\left.554 \mathrm{~nm}\right)$. (c) Chromatograms at different reaction times showing total conversion of the RhB into Rh110 after $240 \mathrm{~min}$. (d) Chromatogram of $\mathrm{RhB}$ after irradiation for $90 \mathrm{~min}$, in which different derivatives of the photodegradation process can be observed. (e) ESI-MS spectra of the species involved in the transformation of RhB into Rh110. (f) Photocatalytic conversion of $\mathrm{EB}$ (blue) and $\mathrm{RhB}(\mathrm{red})$ in the presence of the $\mathrm{Au}-\mathrm{TiO}_{2} \mathrm{NCs}_{\text {(excitation range: } 350-}$ 
$2400 \mathrm{~nm}$ ). (g) Photocatalytic conversion of EB (blue) and RhB (red) in the presence of PS spheres functionalized with $\mathrm{Au}$ NRs and $\mathrm{TiO}_{2}$ NPs (excitation range: 350-2400 nm). (h) Photocatalytic conversion of RhB catalyzed by the $\mathrm{TiO}_{2} \mathrm{NCs}$ (black) and $\mathrm{Au}-\mathrm{TiO}_{2} \mathrm{NCs}$ (red) using the solar simulator (excitation range: $350-2400 \mathrm{~nm}$ ). (i) Photocatalytic conversion of $\mathrm{RhB}$ catalyzed by the $\mathrm{TiO}_{2} \mathrm{NCs}$ (black) and $\mathrm{Au}-\mathrm{TiO}_{2} \mathrm{NCs}$ (red) using the solar simulator with a $700 \mathrm{~nm}$ filter that blocks UV and visible excitations.

The catalytic capsules can also be used for other types of oxidations, such as the transformation of the dihydrorhodamine 123 (dihydro Rh123), a non-fluorescent compound, into its oxidized structure rhodamine 123 (Rh123), a highly fluorescent dye (Figure 3a). Accordingly, we have observed an increase in both the absorption and fluorescence intensities (maxima at 501 and $525 \mathrm{~nm}$, respectively) when dihydro Rh123 is treated with Au$\mathrm{TiO}_{2} \mathrm{NCs}$ under simulated solar irradiation in water (same conditions as those used for the photodegradation of $\mathrm{RhB}$ and EB, but using a filter that blocks $\lambda<700 \mathrm{~nm}$ ) (Figure 3b,c). As validated by LC-MS analyses (Figure S7), such increase is the result of the generation of the fluorescent Rh123 species after conversion of the dihydro Rh123.

Having demonstrated the photocatalytic activity of the $\mathrm{Au}-\mathrm{TiO}_{2} \mathrm{NCs}$ in water, we moved to more complex media in order to assess their ability as bioorthogonal reactors. Therefore, we performed the above photocatalytic transformation of dihydro Rh123 in Dulbecco's modified Eagle's medium (DMEM), a standard milieu used in cell cultures that contains tens of components that range from several inorganic salts, to many amino acids, sugars and vitamins. As shown in Figure 3d, the reaction exhibits a similar profile than in water, thus leading to Rh123 as the product of the photocatalytic transformation. Interestingly, a 10-fold increase in the fluorescence intensity of this molecule can be observed after only 90 minutes of irradiation using just the NIR component of a solar simulator (700-2400 nm). Moreover, such photocatalytic transformation of organic dyes can be similarly carried out in the presence of proteins like BSA (bovine serum albumin protein), which features one free cysteine and several histidine sites in its structure. Accordingly, photodegradation of RhB in the presence of BSA (see Supporting Information for details), using the $\mathrm{Au}-\mathrm{TiO}_{2} \mathrm{NCs}$ as photocatalysts, can be performed using NIR light (Figure S8). As previously discussed, the architecture of the capsule warrants that this type of protein remains 
out of the internal reacting core, which avoids its reaction with ROS generated in this location while preventing damage of the heterogeneous catalytic system.

a<smiles>COC(C)c1ccccc1C1c2ccc(N)cc2Oc2cc(N)ccc21</smiles>

Dihydro Rh123

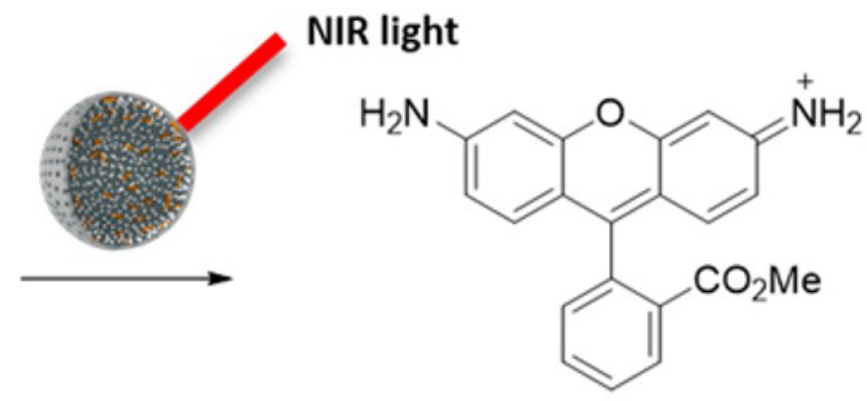

Rh123
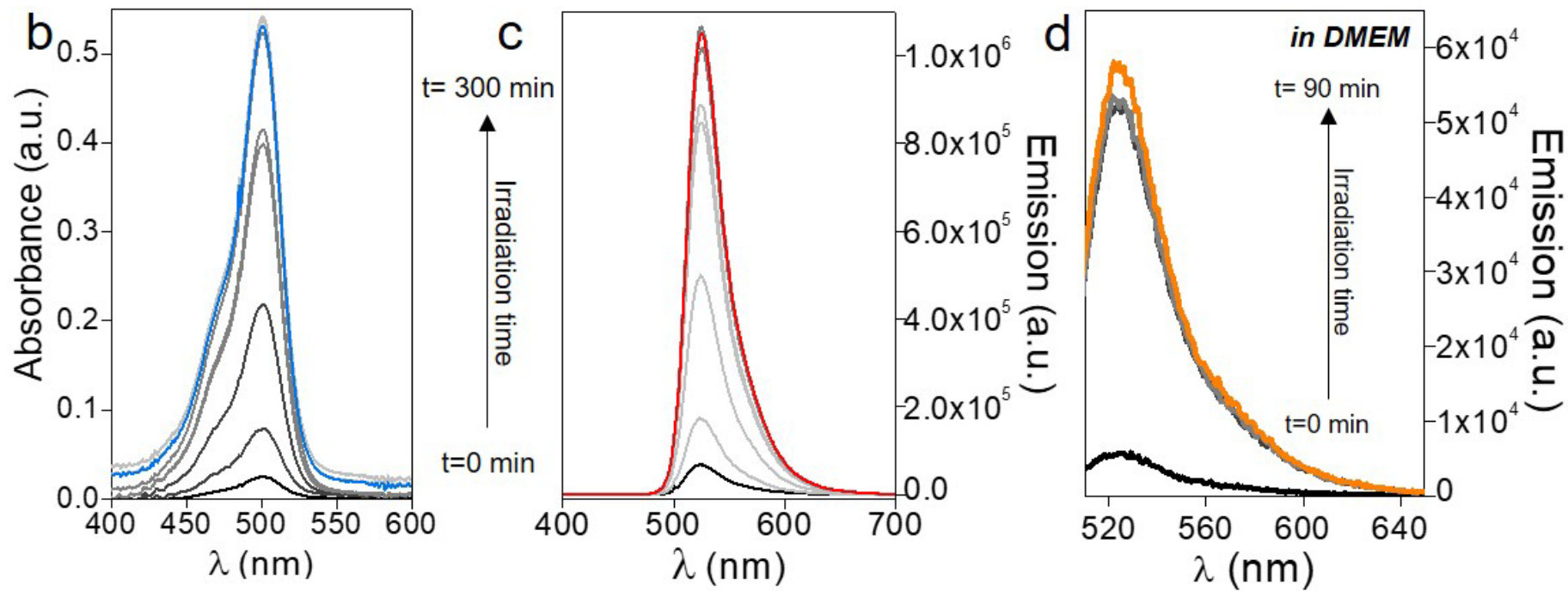

Figure 3. (a) Schematic representation of the photocatalytic oxidation of dihydro Rh123 in the presence of Au$\mathrm{TiO}_{2} \mathrm{NCs}$ with NIR light. (b) Evolution of the UV-Vis absorption spectra of dihydro Rh123 upon irradiation with a solar simulator. (c) Evolution of the fluorescence spectra of Rh123 upon irradiation with the solar simulator $\left(\lambda_{\text {exc }}=500 \mathrm{~nm}\right)$. (d) Evolution of the fluorescence spectra of Rh123 upon irradiation with the solar simulator in $\operatorname{DMEM}\left(\lambda_{\mathrm{exc}}=500 \mathrm{~nm}\right)$. Irradiation range for all the experiments: $700-2400 \mathrm{~nm}$.

As an initial proof of concept to demonstrate the potential of these reactors to achieve bioorthogonal heterogeneous photocatalysis "in living environments", we have carried out preliminary experiments in the presence of living mammalian cells. In this manner, the use of NIR light should ensure biocompatibility while providing for spatiotemporal control in the transformation of an exogenous substrate. Accordingly, incubation of cultured Vero cells with the $\mathrm{Au}-\mathrm{TiO}_{2} \mathrm{NCs}$ for $16 \mathrm{~h}$ allowed the observation of clusters of the hybrid nanoreactors 
in the perinuclear area of the cytosol (yellow circles Figure 4a,b), owing to the endocytic internalization pathway of the catalyst. ${ }^{35}$ Interestingly, no cytotoxic response could be observed at this stage (Figure S9). The medium was then removed and substituted with fresh medium containing $20 \mu \mathrm{M}$ of $\mathrm{RhB}$. After 30 min of incubation, cells were washed again with fresh medium to remove the excess of the fluorescent substrate. Observation under the microscope revealed the accumulation of the fluorescent dye in the aforementioned cluster regions (yellow circle in Figure 4c). Those regions were then irradiated with a $785 \mathrm{~nm}$ laser beam using pulses of 30-60 $\mathrm{s}$ with a spot size of $\approx 2 \mu \mathrm{m}^{2}$. After exposition to the irradiation source a significant decrease in the fluorescence could be observed in these regions (yellow circle in Figure 4d), likely due to the degradative reaction with $\mathrm{RhB}$ (vide supra). Controlling the intensity of the laser beam proved to be crucial in order to avoid undesired cell death from the heating derived from irradiation of the plasmonic NPs. Along these lines, the photocatalytic transformation of the substrate does not lead to any apparent modification of the cellular viability, hence highlighting the biorthogonal capabilities of the hybrid nanoreactor and the confinement of the ROS in its interior (Figure S10).

Control experiments performed using AuNCs led to a negligible decrease in the fluorescent signal (Figure 4e-h) of RhB. This outcome suggests that the local increase in temperature associated to the plasmonic resonators (i.e. plasmonic hyperthermia) does not play a major role in the photocatalytic transformation of the substrate under these experimental conditions. Such result, together with the null effect observed when the same cell line was incubated with $\mathrm{TiO}_{2} \mathrm{NCs}$ (Figure 4i), is consistent with the operation of the hot electron injection process. Therefore, the interaction between the plasmonic component and the semiconductor accounts for the transformation of the chosen substrate in the interior of living cells. 


\section{$\mathrm{Au}-\mathrm{TiO}_{2} \mathrm{NCs}$}
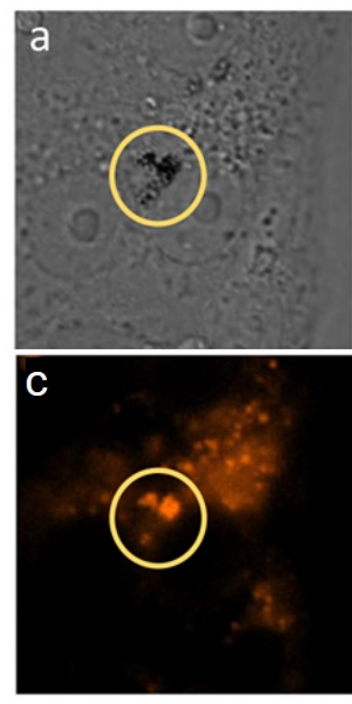

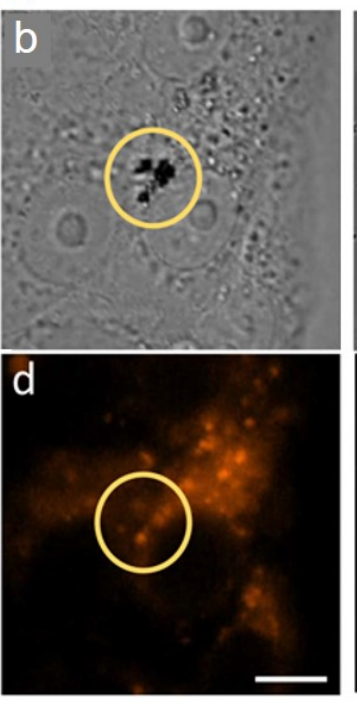

\section{AuNCs}

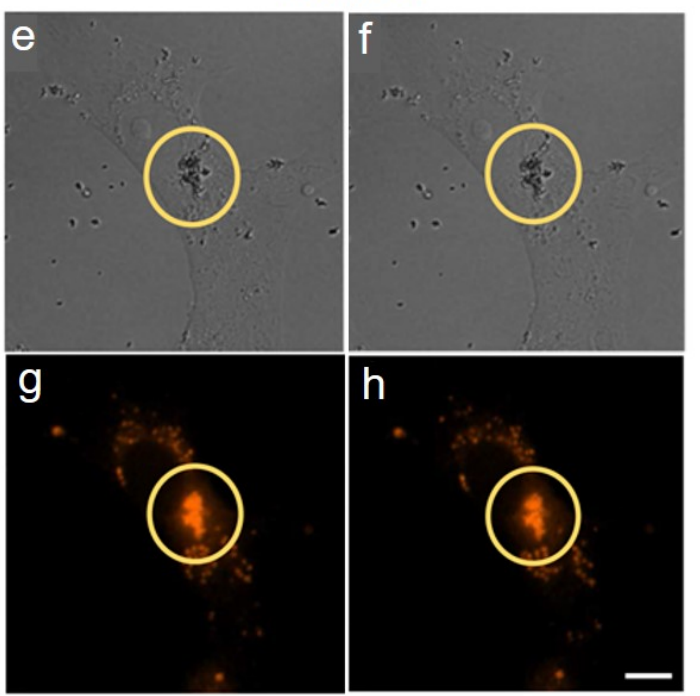

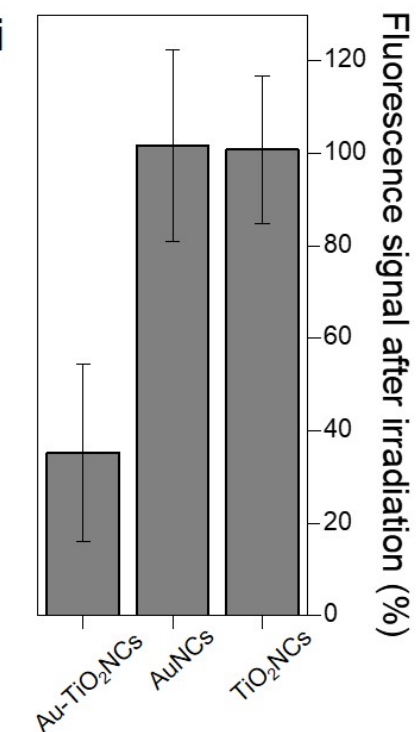

Figure 4. Intracellular decrease in $\mathrm{RhB}$ fluorescence by NIR irradiation of the capsular nanoreactors. Micrographs of Vero cells before $(\mathrm{a}, \mathrm{c})$ and after $(b, \mathrm{~d})$ irradiation in the presence of $\mathrm{Au}-\mathrm{TiO}_{2} \mathrm{NCs}_{\text {(yellow circle) }}$ with a laser beam $(\lambda=785 \mathrm{~nm})$. The same experiments have been repeated in the presence of AuNCs (e-g). The upper panel represents the bright field images showing the morphology of the intracellular NCs while the lower panel corresponds to the fluorescence images showing the intracellular fluorescence $\left(\lambda_{\text {exc }}=543 / 22 \mathrm{~nm}\right.$, $\lambda_{\mathrm{em}}=593 / 40 \mathrm{~nm}$; for more details please refer to the Experimental Section in the SI). Scale bars: $10 \mu \mathrm{m}$. (i) Quantification of the photodegradation experiments with 3 independent silica $\mathrm{NCs}$ : $\mathrm{Au}-\mathrm{TiO}_{2} \mathrm{NCs}, \mathrm{n}=8$; $\mathrm{AuNCs}$, $\mathrm{n}=9$ and $\mathrm{TiO}_{2} \mathrm{NCs}, \mathrm{n}=5$ ( $\mathrm{n}$ refers to the number of experiments performed with each type of $\mathrm{NC}$ ).

In the present study we have described the rational design of remotely activated hybrid heterogeneous photocatalysts engaging the synergistic interaction of a large bandgap semiconductor and a plasmonic resonator, integrated within a dedicated silica nanocapsule. Specifically, $\mathrm{TiO}_{2} \mathrm{NPs}$ and $\mathrm{Au}$ NRs have been immobilized on the internal wall of a hollow mesoporous silica shell to ensure that the catalytic activity is confined within its inner volume. The controlled porosity of the shell allows for a size-selective control of the molecular traffic. These systems have proven to be efficient photocatalysts both in aqueous solutions and in complex biological media, allowing the selective transformation of designed reactants mediated by photogenerated charges. Ultimately, preliminary experiments indicate that the unique morphological features of the nanoreactors allowed 
their use inside cells, for a localized, NIR-promoted degradation of a fluorescent substrate. This work could be viewed among the inaugural contributions of a field at the boundary between heterogeneous photocatalysis and chemical and cell biology.

\section{ASSOCIATED CONTENT}

The Supporting Information is available free of charge on the ACS Publications website at DOI: XXXX

Experimental methods; HRTEM and TEM characterization; chemical structures; chromatograms and ESI-MS spectra; control photocatalytic experiments and toxicity assays.

\section{AUTHOR INFORMATION}

Corresponding authors

*E-mail: miguel.comesana-hermo@u-paris.fr

*E-mail: joseluis.mascarenas@usc.es

*E-mail: macorrea@uvigo.es

\section{ORCID}

Ana Sousa-Castillo: 0000-0001-7811-0646

José R. Couceiro: 0000-0002-5742-1071

María Tomás-Gamasa: 0000-0001-8681-2744

Andrea Mariño-López: 0000-0002-5745-3030

Fernando López: 0000-0002-0235-6858

Walid Baaziz: 0000-0002-3749-6105

Ovidiu Ersen: 0000-0002-1553-0915

Miguel Comesaña-Hermo: 0000-0001-8471-5510

José L. Mascareñas: 0000-0002-7789-700X

Miguel A. Correa-Duarte: 0000-0003-1950-1414

\section{NOTES}

The authors declare no competing financial interest.

\section{ACKNOWLEDGMENTS}

This work was supported by the Ministerio de Economía y Competitividad (MINECO, Spain) under the Grant (CTM2017-84050-R, SAF2016-76689-R), Xunta de Galicia/FEDER (2015-CP082, ED431C 2017/19, IN607A 
2018/5 and Centro Singular de Investigación de Galicia accreditation 2019-2022, ED431G 2019/06 and ED431G 2019/09), 0245_IBEROS_1_E, 0712_ACUINANO_1_E, 0624_2IQBIONEURO_6_E and NANOCULTURE cofounded by FEDER through the program Interreg V-A España-Portugal (POCTEP), NANOCULTURE (ERDF: 1.102.531) Interreg Atlantic Area, the European Union (European Regional Development Fund-ERDF) and the European Research Council (Advanced Grant No. 340055). M. T. G thanks the financial support from the Agencia Estatal de Investigación (RTI2018-093813-J-I00).

\section{BIBLIOGRAPHY}

(1) Fujishima, A.; Zhang, X.; Tryk, D. A. TiO2 Photocatalysis and Related Surface Phenomena. Surf. Sci. Rep. 2008, 63, 515-582.

(2) Lewicka, Z. A.; Yu, W. W.; Oliva, B. L.; Contreras, E. Q.; Colvin, V. L. Photochemical Behavior of Nanoscale TiO2 and ZnO Sunscreen Ingredients. J. Photochem. Photobiol. A Chem. 2013, 263, $24-33$.

(3) Rehman, F. U.; Zhao, C.; Jiang, H.; Wang, X. Biomedical Applications of Nano-Titania in Theranostics and Photodynamic Therapy. Biomater. Sci. 2016, 4, 40-54.

(4) Meng, L.; Chen, Z.; Ma, Z.; He, S.; Hou, Y.; Li, H. H.; Yuan, R.; Huang, X. H.; Wang, X.; Wang, X.; Long, J. Gold Plasmon-Induced Photocatalytic Dehydrogenative Coupling of Methane to Ethane on Polar Oxide Surfaces. Energy Environ. Sci. 2018, 11, 294-298.

(5) Liu, J.; Feng, J.; Gui, J.; Chen, T.; Xu, M.; Wang, H.; Dong, H.; Chen, H.; Li, X.; Wang, L.; et al. Metal@semiconductor Core-Shell Nanocrystals with Atomically Organized Interfaces for Efficient Hot Electron-Mediated Photocatalysis. Nano Energy 2018, 48, 44-52.

(6) Zhang, Y.; He, S.; Guo, W.; Hu, Y.; Huang, J.; Mulcahy, J. R.; Wei, W. D. Surface-Plasmon-Driven Hot Electron Photochemistry. Chem. Rev. 2018, 118, 2927-2954.

(7) Wang, S.; Gao, Y.; Miao, S.; Liu, T.; Mu, L.; Li, R.; Fan, F.; Li, C. Positioning the Water Oxidation Reaction Sites in Plasmonic Photocatalysts. J. Am. Chem. Soc. 2017, 139, 11771-11778. 
Negrín-Montecelo, Y.; Comesaña-Hermo, M.; Khorashad, L. K.; Sousa-Castillo, A.; Wang, Z.; PérezLorenzo, M.; Liedl, T.; Govorov, A. O.; Correa-Duarte, M. A. Photophysical Effects behind the Efficiency of Hot Electron Injection in Plasmon-Assisted Catalysis: The Joint Role of Morphology and Composition. ACS Energy Lett. 2020, 5, 395-402.

(9) Robatjazi, H.; Zhao, H.; Swearer, D. F.; Hogan, N. J.; Zhou, L.; Alabastri, A.; McClain, M. J.; Nordlander, P.; Halas, N. J. Plasmon-Induced Selective Carbon Dioxide Conversion on Earth-Abundant Aluminum-Cuprous Oxide Antenna-Reactor Nanoparticles. Nat. Commun. 2017, 8, 1-9.

(10) Tsukamoto, D.; Shiraishi, Y.; Sugano, Y.; Ichikawa, S.; Tanaka, S.; Hirai, T. Gold Nanoparticles Located at the Interface of Anatase/Rutile TiO2 Particles as Active Plasmonic Photocatalysts for Aerobic Oxidation. J. Am. Chem. Soc. 2012, 134, 6309-6315.

(11) Tomás-Gamasa, M.; Mascareñas, J. L. TiO2-Based Photocatalysis at the Interface with Biology and Biomedicine. ChemBioChem 2020, 21, 294-309.

(12) Sletten, E. M.; Bertozzi, C. R. From Mechanism to Mouse: A Tale of Two Bioorthogonal Reactions. Acc. Chem. Res. 2011, 44, 666-676.

(13) Li, J.; Kong, H.; Zhu, C.; Zhang, Y. Photo-Controllable Bioorthogonal Chemistry for Spatiotemporal Control of Bio-Targets in Living Systems. Chem. Sci. 2020, 11, 3390-3396.

(14) Kumar, A.; Kumar, S.; Kumari, N.; Lee, S. H.; Han, J.; Michael, I. J.; Cho, Y. K.; Lee, I. S. Plasmonically Coupled Nanoreactors for NIR-Light-Mediated Remote Stimulation of Catalysis in Living Cells. ACS Catal. 2019, 9, 977-990.

(15) Espinosa, A.; Kolosnjaj-Tabi, J.; Abou-Hassan, A.; Plan Sangnier, A.; Curcio, A.; Silva, A. K. A.; Di Corato, R.; Neveu, S.; Pellegrino, T.; Liz-Marzán, L. M.; Wilhelm, C. Magnetic (Hyper)Thermia or Photothermia? Progressive Comparison of Iron Oxide and Gold Nanoparticles Heating in Water, in Cells, and In Vivo. Adv. Funct. Mater. 2018, 28, 1-16. 
(16) Rivera-Gil, P.; Vazquez-Vazquez, C.; Giannini, V.; Callao, M. P.; Parak, W. J.; Correa-Duarte, M. A.; Alvarez-Puebla, R. A. Plasmonic Nanoprobes for Real-Time Optical Monitoring of Nitric Oxide inside Living Cells. Angew. Chem. Int. Ed. 2013, 52, 13694-13698.

(17) Destito, P.; Sousa-Castillo, A.; Couceiro, J. R.; López, F.; Correa-Duarte, M. A.; Mascareñas, J. L. Hollow Nanoreactors for Pd-Catalyzed Suzuki-Miyaura Coupling and O-Propargyl Cleavage Reactions in Bio-Relevant Aqueous Media. Chem. Sci. 2019, 10, 2598-2603.

(18) Sousa-Castillo, A.; Comesaña-Hermo, M.; Rodríguez-González, B.; Pérez-Lorenzo, M.; Wang, Z.; Kong, X.-T.; Govorov, A. O.; Correa-Duarte, M. A. Boosting Hot Electron-Driven Photocatalysis through Anisotropic Plasmonic Nanoparticles with Hot Spots in Au-TiO2 Nanoarchitectures. J. Phys. Chem. C 2016, 120, 11690-11699.

(19) Ingram, D. B.; Christopher, P.; Bauer, J. L.; Linic, S. Predictive Model for the Design of Plasmonic Metal/Semiconductor Composite Photocatalysts. ACS Catal. 2011, 1, 1441-1447.

(20) Liz-Marzán, L. M. Tailoring Surface Plasmons through the Morphology and Assembly of Metal Nanoparticles. Langmuir 2006, 22, 32-41.

(21) Tsai, M.-F.; Chang, S.-H. G.; Cheng, F.-Y.; Shanmugam, V.; Cheng, Y.-S.; Su, C.-H.; Yeh, C.-S. Au Nanorod Design as Light-Absorber in the First and Second Biological Near-Infrared Windows for in Vivo Photothermal Therapy. ACS Nano 2013, 7, 5330-5342.

(22) Carrillo-Carrión, C.; Martínez, R.; Navarro Poupard, M. F.; Pelaz, B.; Polo, E.; Arenas-Vivo, A.; Olgiati, A.; Taboada, P.; Soliman, M. G.; Catalán, Ú.; Fernández-Castillejo, S.; Solà, R.; Parak, W. J.; Horcajada, P.; Alvarez-Puebla, R. A.; del Pino, P. Aqueous Stable Gold Nanostar/ZIF-8 Nanocomposites for LightTriggered Release of Active Cargo Inside Living Cells. Angew. Chem. Int. Ed. 2019, 58, 7078-7082.

(23) Hembury, M.; Chiappini, C.; Bertazzo, S.; Kalber, T. L.; Drisko, G. L.; Ogunlade, O.; Walker-Samuel, S.; Krishna, K. S.; Jumeaux, C.; Beard, P.; Kumar, C. S. S. R.; Porter, A. E.; Lythgoe, M. F.; Boissière, 
C.; Sanchez, C.; Stevens, M. M. Gold-Silica Quantum Rattles for Multimodal Imaging and Therapy.

Proc. Natl. Acad. Sci. U. S. A. 2015, 112, 1959-1964.

(24) Pelaz, B.; Alexiou, C.; Alvarez-Puebla, R. A.; Alves, F.; Andrews, A. M.; Ashraf, S.; Balogh, L. P.; Ballerini, L.; Bestetti, A.; Brendel, C.; et al. Diverse Applications of Nanomedicine. ACS Nano 2017, 11, 2313-2381.

(25) Inumaru, K.; Yasui, M.; Kasahara, T.; Yamaguchi, K.; YAsuda, A.; Yamanaka, S. Nanocomposites of Crystalline TiO2 Particles and Mesoporous Silica : Molecular Selective Photocatalysis Tuned by Controlling Pore Size and Structure. J. Mater. Chem. 2011, 21, 12117-12125.

(26) Chen, Y.; Chen, H.; Shi, J. In Vivo Bio-Safety Evaluations and Diagnostic/Therapeutic Applications of Chemically Designed Mesoporous Silica Nanoparticles. Adv. Mater. 2013, 25, 3144-3176.

(27) Argyo, C.; Weiss, V.; Bräuchle, C.; Bein, T. Multifunctional Mesoporous Silica Nanoparticles as a Universal Platform for Drug Delivery. Chem. Mater. 2014, 26, 435-451.

(28) Cavigli, L.; Cini, A.; Centi, S.; Borri, C.; Lai, S.; Ratto, F.; de Angelis, M.; Pini, R.; Pini, R. Photostability of Gold Nanorods upon Endosomal Confinement in Cultured Cells. J. Phys. Chem. C 2017, 121, 6393-6400.

(29) Vázquez-Vázquez, C.; Vaz, B.; Giannini, V.; Pérez-Lorenzo, M.; Álvarez-Puebla, R. A.; Correa-Duarte, M. A. Nanoreactors for Simultaneous Remote Thermal Activation and Optical Monitoring of Chemical Reactions. J. Am. Chem. Soc. 2013, 135, 13616-13619.

(30) Caruso, F.; Caruso, R. A.; Möhwald, H. Nanoengineering of Inorganic and Hybrid Hollow Spheres by Colloidal Templating. Science 1998, 282, 1111-1114.

(31) Natarajan, T. S.; Thomas, M.; Natarajan, K.; Bajaj, H. C.; Tayade, R. J. Study on UV-LED/TiO2 Process for Degradation of Rhodamine B Dye. Chem. Eng. J. 2011, 169, 126-134. 
(32) Wu, T.; Liu, G.; Zhao, J.; Hidaka, H.; Serpone, N. Photoassisted Degradation of Dye Pollutants . V . Self-Photosensitized Oxidative Transformation of Rhodamine B under Visible Light Irradiation in Aqueous TiO2 Dispersions. J. Phys. Chem. B 1998, 102, 5845-5851.

(33) Ayodhya, D.; Veerabhadram, G. A Review on Recent Advances in Photodegradation of Dyes Using Doped and Heterojunction Based Semiconductor Metal Sulfide Nanostructures for Environmental Protection. Mater. Today Energy 2018, 9, 83-113.

(34) Alberti, S.; Soler-Illia, G. J. A. A.; Azzaroni, O. Gated Supramolecular Chemistry in Hybrid Mesoporous Silica Nanoarchitectures: Controlled Delivery and Molecular Transport in Response to Chemical, Physical and Biological Stimuli. Chem. Commun. 2015, 51, 6050-6075.

(35) Iversen, T. G.; Skotland, T.; Sandvig, K. Endocytosis and Intracellular Transport of Nanoparticles: Present Knowledge and Need for Future Studies. Nano Today 2011, 6, 176-185. 
TOC

Remote Activation of Hollow Nanoreactors for Heterogeneous

Photocatalysts in Bio-relevant Media

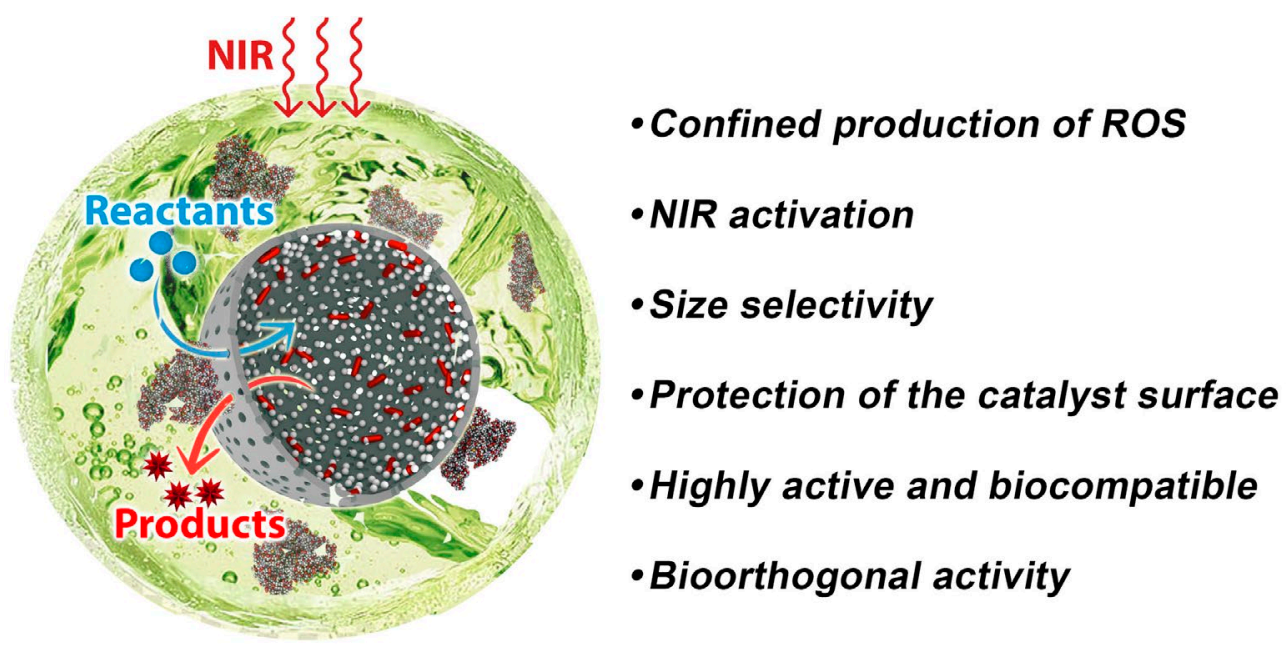

